\title{
Scalar gradient behaviour in MILD combustion
}

\author{
Y. Minamoto, N. Swaminathan \\ Department of Engineering, Cambridge University, Cambridge, CB2 1PZ, UK
}

\begin{abstract}
The results of three-dimensional Direct Numerical Simulation (DNS) of Moderate, Intense Low-oxygen Dilution (MILD) and conventional premixed turbulent combustion conducted using a skeletal mechanism including the effects of nonunity Lewis numbers and temperature dependent transport properties are analysed to investigate combustion characteristics using scalar gradient information. The DNS data is also used to synthesise laser induced fluorescence (LIF) signals of $\mathrm{OH}, \mathrm{CH}_{2} \mathrm{O}$, and $\mathrm{CHO}$. These signals are analysed to verify if they can be used to study turbulent MILD combustion and it has been observed that at least two $\left(\mathrm{OH}\right.$ and $\mathrm{CH}_{2} \mathrm{O}$ ) LIF signals are required since the $\mathrm{OH}$ increase across the reaction zone is smaller in MILD combustion compared to premixed combustion. The scalar gradient PDFs conditioned on the reaction rate obtained from the DNS data and synthesised LIF signals suggests a strong gradient in the direction normal to the MILD reaction zone with moderate reaction rate implying flamelet combustion. However, the PDF of the normal gradient is as broad as for the tangential gradient when the reaction rate is high. This suggests a non-flamelet behaviour, which is due to interaction of reaction zones. The analysis of the conditional PDFs for the premixed case confirms the expected behaviour of scalar gradient in flamelet combustion. It has been shown that the LIF signals synthesised using 2D slices of DNS data also provide very similar insights. These results demonstrate
\end{abstract}


that the so-called flameless combustion is not an idealised homogeneous reactive mixture but has common features of conventional combustion while containing distinctive characteristics.

Keywords: MILD combustion, flameless combustion, Scalar gradient, Direct numerical simulation (DNS), LIF

\section{Introduction}

Combustion systems must be improved constantly to achieve high efficiency and reduced emission simultaneously to meet the ever stringent emission legislation and environmental requirements. A number of approaches are being explored to meet these requirements. Although fuel lean premixed combustion is one of the potential methods for clean combustion, ignitability and instability of lean combustion become significant issues to address. However, some of these can be avoided by preheating the reactant mixture using the heat in the exhaust stream. The preheating results in higher flame temperature which can cause the thermal $\mathrm{NO}_{x}$ to increase if there is substantial level of oxygen in the reactant stream. The thermal $\mathrm{NO}_{x}$ formation can also be reduced by using the exhaust gas recirculation (EGR) techniques. The recirculation can be either internal or external to the combustor and external EGR is common in heavy duty diesel engines and internal recirculation is common in furnaces. At very high EGR rate, the oxygen available for combustion is reduced to a very low level (typically lower than $5 \%$ by volume) and combustion under these conditions is known as MILD (moderate or intense low-oxygen dilution) combustion, which is also commonly known as flameless combustion. The internal recirculation is commonly used to achieve the MILD combustion. 
The MILD combustion technology [1, 2, 3] has potentials to achieve high reactant temperature and low temperature rise across the combustion zone, and thereby resulting in desirable combustion characteristics. The standard definition of MILD combustion is as follows. The reactant temperature, $T_{r}$, is higher than the autoignition temperature, $T_{i g n}$, of a given mixture and the temperature rise during combustion, $\Delta T=T_{p}-T_{r}$, is smaller than $T_{i g n}$. The symbol $T_{p}$ denotes the product temperature. In order to meet $\Delta T<T_{i g n}$, the reactant mixture is diluted highly with exhaust gas so that the oxygen content in the reactant mixture is typically about 2 to $5 \%$ by volume [3]. The combustion efficiency is enhanced because of the elevated reactant temperature $[1,2,3]$ and the thermal $\mathrm{NO}_{x}$ formation is suppressed significantly because of low oxygen concentration and low combustion temperature resulting from high dilution $[1,2,3]$. Typically, it takes about few seconds to produce substantial level of thermal $\mathrm{NO}_{x}$ at around $1900 \mathrm{~K}$ and this reduces to few milliseconds at about $2300 \mathrm{~K}$ [1]. The peak temperature in the MILD combustion is typically less than $1900 \mathrm{~K}$. Also, the combustion noise and instabilities are reduced because of low temperature rise across the combustion zone $[1,2,3]$. These advantages make the MILD combustion as a potential candidate for environmentally friendlier thermal power generation. However, our fundamental understanding of this combustion is not complete yet.

Laser diagnostics using OH-PLIF [4, 5, 6, 7] suggested the presence of thin reaction zones in MILD combustion. A recent DNS study [8] supported this and showed an abundance of interacting flamelets in the MILD turbulent combustion. In addition to these interactions, it has also been observed that propagating flames and autoignition are present.The OH-PLIF is commonly used in laser diagnostics of chemically reacting turbulent flows because of its high concentration and well 
understood spectroscopy characteristics [9]. However, using OH-PLIF alone may be insufficient to investigate turbulent MILD combustion reaction zones because of their characteristics described above and the presence of $\mathrm{OH}$ in the unreacted mixture which has been diluted with exhaust gases. Furthermore, the $\mathrm{OH}$ level inside these zones may not be significantly larger than that in the unburnt or burnt regions, especially when the oxygen level is near the lower end for the MILD combustion. This issue may be addressed through careful calibration of the $\mathrm{OH}-$ LIF system. However, a second marker would be required to identify the MILD reaction zones unambiguously.

Many previous studies $[10,11,12,13,14,15,16,17,18]$ on turbulent premixed flames used PLIF signal of particular species such as $\mathrm{CH}$ and $\mathrm{CHO}$, and the products of PLIF signals of particular species such as $S_{\mathrm{OH}} \times S_{\mathrm{CH}_{2} \mathrm{O}}$ to identify heat releasing regions. The choice of these scalars are fuel mixture specific $[11,19,20]$ and, they have been tested and used for stoichiometric and lean premixed methane-air flames in the past. For example, the marker $\mathrm{CH}$ has reasonable correlation with heat release only for undiluted reactant mixture with equivalence ratio of around 0.8-1.2 [15]. Thus, this marker may not be a suitable choice for the MILD combustion. The signal-to-noise ratio for CHO-PLIF imaging is generally low compared to $\mathrm{OH}$ and formaldehyde $[11,14]$ although it is possible to get good signal for $\mathrm{CHO}$ imaging using multimode lasers [15].

The PLIF measurements of $\mathrm{OH}$ and $\mathrm{CH}_{2} \mathrm{O}$ have been conducted for MILD combustion in past studies $[7,21]$. However, thorough and direct investigation into the applicability of these chemical markers to estimate heat release rate has not been carried out for MILD combustion. Thus, it is useful to test if the above conventional markers to identify heat releasing zones work for the MILD combus- 
tion also. This is the prime motive for this study. The representative PLIF signals of the markers $\mathrm{OH}, \mathrm{CH}_{2} \mathrm{O}$ and $\mathrm{CHO}$ are constructed using direct numerical simulation (DNS) data of turbulent MILD combustion first. These signals are then used to evaluate the adequacy of these markers by comparing the reconstructed heat releasing regions with those from the DNS data. The third objective of this study is to investigate the behaviour of a reaction progress variable gradient obtained from the DNS data and the constructed PLIF images. Although the onset of turbulent MILD combustion from initial mixture can be studied using DNS, as has been done in $[22,23]$ for laminar conditions, it is not the focus of this study and this will be addressed in future.

This paper is organised as follows. The DNS of the MILD combustion is described in the next section along with the data processing techniques used in this study. The premixing used in this study represents the turbulent mixing of fuel, air and recirculated exhausts stream inside a MILD combustor that can occur before a flame or an autoignition front is established. More detail on this is given in sections 2.1 and 2.2. The results on the reaction zones and reaction progress variable are discussed in section 3. The scalar gradients are studied in section 4 . The conclusions are summarised in the final section.

\section{DNS of MILD and conventional premixed combustion}

\subsection{Flow configuration and numerical method}

The configurations such as EGR, flue gas recirculation (FGR) and staged fuel injection are used to achieve MILD combustion in practice by injecting fuel and air into a stream of hot products. Although the high momentum jet typically used in MILD combustors can enhance mixing, a limited time available for this mix- 
ing may result in a spatially and temporally inhomogeneous mixture consisting of fresh and recirculated exhaust gases. The sizes of these pockets are random and determined by the turbulence conditions. This inhomogeneous mixture either autoignites or establishes a flame depending on the local turbulence and thermochemical conditions. To represent this scenario, a non-uniform mixture field consisting of fresh and exhaust gases is constructed carefully to mimic the mixing processes described above and to study the evolution of MILD reaction zones under non-uniformly mixed charge conditions. The steps involved in generating this inhomogeneous field are described in section 2.2. Detailed classifications of various MILD combustion configurations in a mixing layer can be found in previous studies $[24,25,26]$.

The computational domain has a reflecting inflow and a non-reflecting outflow boundary specified using the Navier-Stokes Characteristic Boundary Conditions (NSCBC) formulation [27, 28] with Local One-Dimensional Inviscid (LODI) approximation in the $x$ direction and periodic conditions in the $y$ and $z$ directions. A similar computational domain with these boundary conditions is also used for a conventional turbulent lean premixed flame case. The schematic of these computational domains are shown in Fig. 1, which will be discussed later in Section 3.

The numerical code SENGA2 [29], an updated version of SENGA [28], used in earlier investigations of turbulent premixed flames [30,31] and MILD combustion [8] is employed for this study. This code solves fully compressible transport equations on a uniform mesh for mass, momentum, internal energy and scalar mass fractions along with temperature dependent transport properties. The spatial derivatives are obtained using a tenth order central difference scheme which gradually reduces to a fourth order scheme near boundaries. The integration in time is 


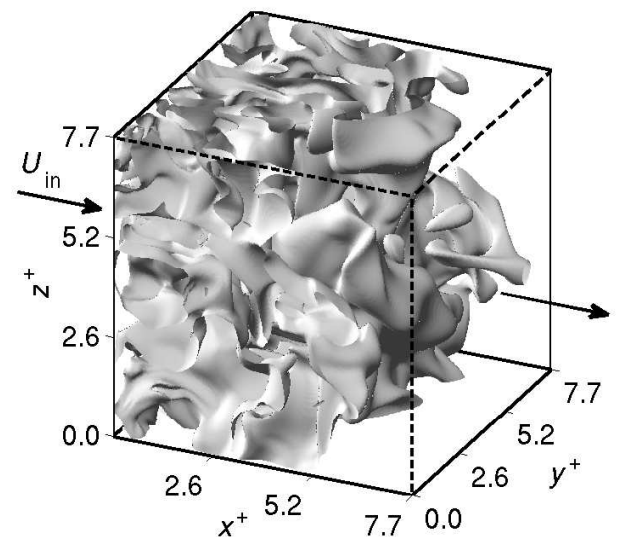

(a)

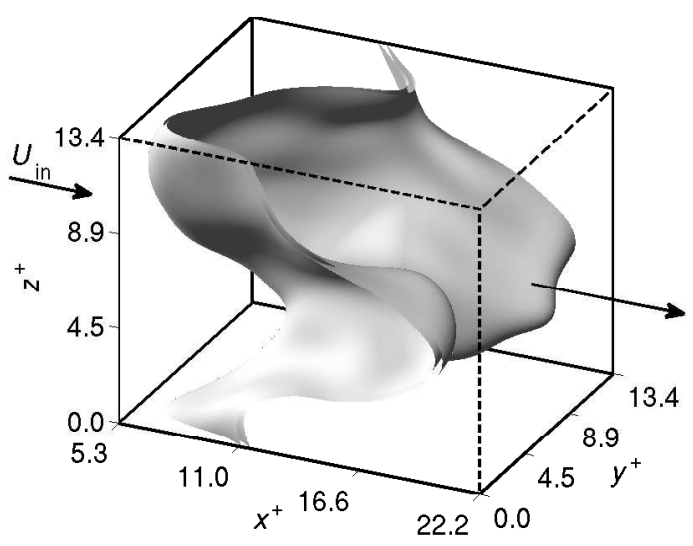

(b)

Figure 1: Computational domain for (a) MILD and (b) conventional premixed combustion. The reaction rate iso-surface for $\omega_{c_{T}}^{+}=2.0$ is shown for Case B in (a) and $\omega_{c_{T}}^{+}=1.0$ for Case $\mathrm{C}$ in (b). Note that only $5.3 \leq x^{+} \leq 22.2$ of the domain is shown for Case $\mathrm{C}$. Both snapshots are taken at $t=1.5 \tau_{D}$.

achieved using a third order Runge-Kutta scheme, although a fourth order scheme can be used in SENGA2. The numerical stability of these schemes is maintained by using sufficiently small time steps ( $\Delta t \leq 1 \times 10^{-10} \mathrm{~s}$ ), which is dictated by the acoustic CFL condition. The methane-air combustion is simulated using a skeletal mechanism involving 16 species and 36 elementary reactions [32].

An inhomogeneous mixture containing pockets of unburnt and burnt gases flows at an average velocity of $U_{\text {in }}$ through the inflow boundary located at $x=0$ of the computational domain for MILD combustion cases as shown in Fig. 1. The inflowing fields of scalar mass fractions, $Y_{i}(x=0, y, z, t)$, temperature, $T(x=0, y, z, t)$, and velocity, $\boldsymbol{u}(x=0, y, z, t)$, are specified using preprocessed fields $\hat{Y}_{i}[\underline{x}(t), y, z], \hat{T}[\underline{x}(t), y, z]$ and $\hat{\boldsymbol{u}}[\underline{x}(t), y, z]$, where $\underline{x}(t)$ denotes the $x$ location of a scanning plane at time $t$ moving at a velocity of $U_{i n}$ through the prepro- 


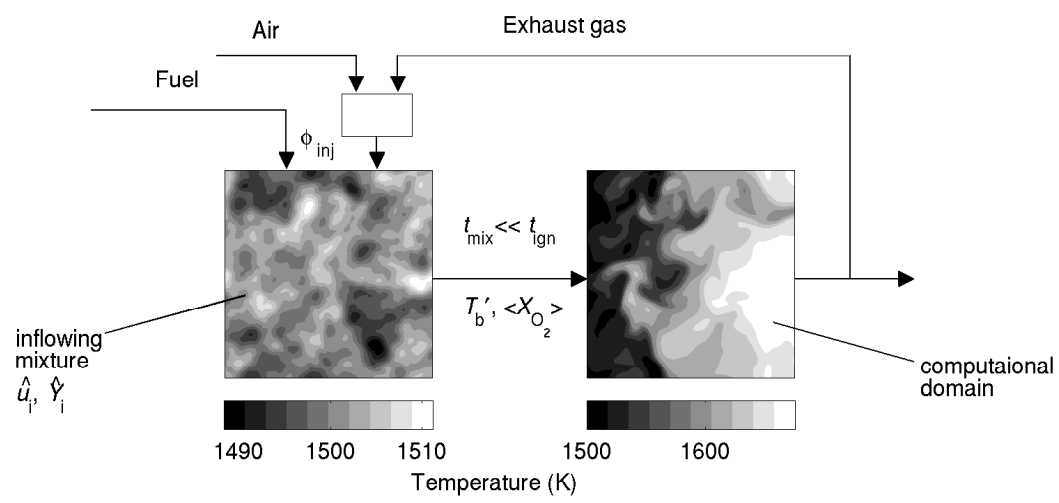

Figure 2: Schematic illustration of an EGR type combustion.

cessed fields, which are constructed as described in section 2.2. For a conventional premixed case, only a turbulence field $\hat{\boldsymbol{u}}[\underline{x}(t), y, z]$ is preprocessed and fed to the computational domain from the inlet boundary at an average velocity of $U_{i n}$.

\subsection{Construction of initial and inflow fields}

Direct numerical simulation of a complete MILD combustion system is not feasible yet because of heavy computational cost involved. So, the simulation is split into two phases to mimic the physical processes of MILD combustion as noted in section 2.1. The first phase involves the generation of a non-uniform and inhomogeneous mixture field which is consistent with the required turbulence and combustion conditions, and the second phase is the turbulent MILD combustion. These two phases are represented pictorially in Fig. 2. The desired inhomogeneous fields of $\hat{\boldsymbol{u}}$ and $\hat{Y}_{i}$ are obtained following the steps in [8], which are described below for the sake of completeness.

Step (i) A turbulent velocity field is simulated in a preliminary DNS of freely decaying homogeneous isotropic turbulence inside a periodic cubic domain for each combustion DNS case. This flow field is first initialised 
using a turbulent kinetic energy spectrum [33] as described by [34]. After this initialisation, the simulation is continued until the velocity derivative skewness reaches an approximately a constant value of -0.5 representing a fully developed turbulence field and desirable turbulence intensity is achieved.

Step (ii) One-dimensional laminar flames freely propagating into reactant mixtures of a desired MILD conditions are calculated using the skeletal mechanism of [32]. The reactants for this laminar flames is diluted with products of fully burnt mixture $\left(X_{\mathrm{H}_{2} \mathrm{O}}: X_{\mathrm{CO}_{2}}=2: 1\right)$, and the molar fraction of $\mathrm{O}_{2}$ in the reactant mixture $X_{\mathrm{O}_{2}, r}$ is matched the desired dilution level.

Step (iii) An initial homogeneous scalar field is obtained by specifying a scalarenergy spectrum function as in [35]. This scalar field is taken as an initial field of a reaction progress variable, defined as $c_{Y}=1-Y_{f} / Y_{f, r}$ taking values between 0 to 1 . The same initial $c_{Y}$ field is used to initialise all the MILD combustion simulations conducted in this study. The fuel mass fraction is $Y_{f}$ and the subscript, $r$, denotes reactant mixture. The flame solution from Step (ii) is then mapped to this $c_{Y}$ field to obtain the spatial variation of $Y_{\alpha}$. The temperature is set to a constant value of $T_{m}$ to be specified later. Such nearly-constant temperature and fluctuating species fields before combustion occur may be observed in MILD combustor employing recuperative or regenerative heat exchangers to heat up a mixture of fuel, air and exhaust gases [4]. The fluctuating scalar field obtained as above do not yet have any cor- 
relation with the turbulence field obtained in Step (i).

Step (iv) These scalar and turbulence fields are then allowed to evolve in the periodic domain to mimic the EGR-mixing without any chemical reaction. This mixing DNS is run for one large eddy time, $\ell_{0} / v^{\prime}$, which is much shorter than the autoignition delay time for the chosen mixture. This allows the correlation between the turbulence and scalar fields to develop. The root-mean-square (RMS) of the turbulent fluctuations obtained in Step (i) is $v^{\prime}$ and its integral length scale is $\ell_{0}$. The internal energy equation is also solved in this step to mimic the evolution of temperature during the mixing process, which results in a maximum temperature fluctuation of about $2 \%$ of the mean value, $T_{m}$.

The Bilger's mixture fraction [36] in the initial mixture field generated as described above has a variation of about $\pm 5 \%$ of the mean value, $\langle\xi\rangle$, which results from the inhomogeneity in the field and difference in the mass diffusivity of the various species. The equivalence ratio obtained using $\phi=\left(1-\xi_{s t}\right) \xi /(1-\xi) \xi_{s t}$, where $\xi_{s t}$ is the stoichiometric mixture fraction, gives a mean value of $\langle\phi\rangle=0.8$ for all the cases considered in this study. The calculation of the mixture fraction is based on the boundary condition for the air stream diluted with products to a desired level of oxygen and a pure fuel stream as shown in Fig. 2.

\subsection{Numerical conditions}

The velocity and scalar fields obtained as described in Section 2.2 are used as the initial and inflow fields in the DNS of MILD combustion. Two cases having MILD combustion conditions (Cases A and B) and one conventional premixed combustion case (Case C) are considered. The initial/inlet mixture for Case A is 

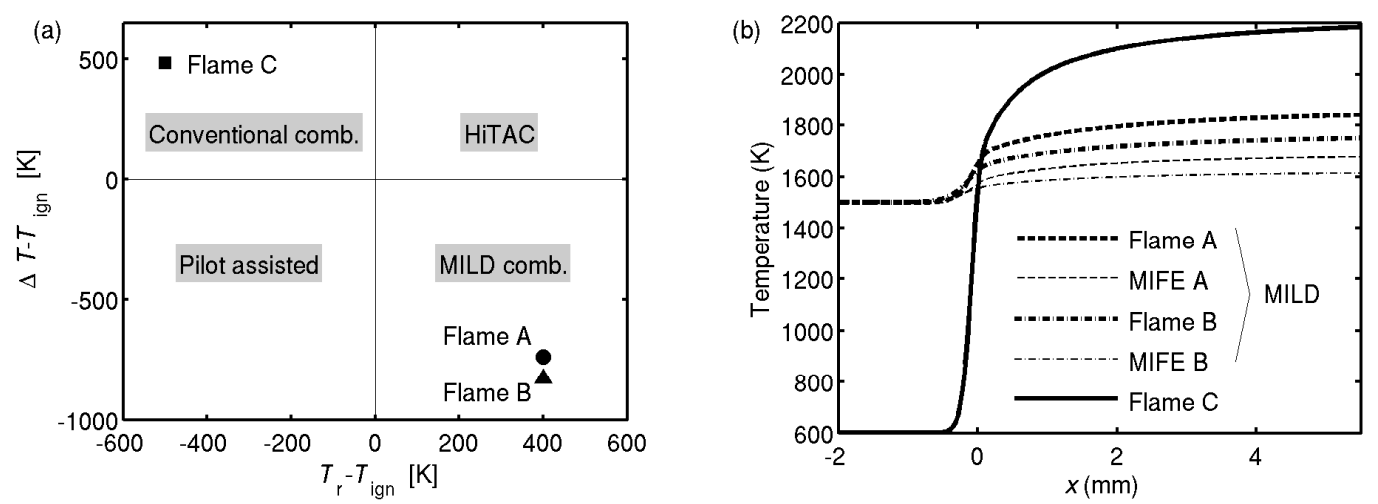

Figure 3: A diagram showing combustion types [3] (a) and temperature variations in laminar MILD and conventional flames $(x=0$ is the peak location of the heat release rate) (b).

constructed based on a 1D laminar flame, Flame A, and that for Case B is based on Flame B. The initial field for the conventional turbulent premixed flame, Case C, is based on Flame C. These cases are shown in a combustion type diagram in Fig. 3, and their thermochemical conditions are given in Table 1. These mixtures do yield propagating flame solutions and thus the standard combustion characteristics such as flame speed, flame thickness and ignition delay time can be computed for these mixtures. These values are given in Table 1 along with product temperature and mole fractions of various species in the reactant mixture. The laminar flame speed is $S_{L}$ and the Zeldovich thickness is $\delta_{F}=\alpha / S_{L}$, where $\alpha$ is the thermal diffusivity of the mixture. These flame quantities are computed using 1D version of SENGA2 and these values are close to those obtained using the PREMIX code [37]. When the reactant temperature is larger than its autoignition temperature the value of $S_{L}$ may depend on the computational domain size and the values in Table 1 are computed using a domain size of $10 \mathrm{~mm}$, which is the largest length employed for 
the DNS computations (see later part of this section). Since $S_{L}$ is not unique [38] for $T_{r} \geq T_{i g n}$, retaining the computational domain size allows one to have a basis for a meaningful analyses. For these reasons, the $S_{L}$ given in Table 1 for reactants with $T_{r} \geq T_{i g n}$ must be read with caution. The autoignition delay time, $\tau_{i g n}$, given in Table 1 is computed using a zero-dimensional, constant pressure wellstirred reactor (WSR) model available in Chemkin package [37] and employing the maximum temperature gradient criterion. The presence of turbulence can alter the balance among unsteady evolution, reaction and diffusion processes locally leading to a change in ignition delay time compared to the WSR model value. This is not considered here and thus the value of $\tau_{i g n}$ given in Table 1 is only for a guidance purpose and one must use a partially stirred reactor (PaSR) model to include turbulence effects. The PaSR will obviously introduce some uncertainties due to modelling involved in that approach.

Although the initial mixture field for the MILD cases are constructed based on either the Flame A or Flame B, suitable representative one-dimensional laminar flames for these cases were found to be those having reactant mixture composition for major species equal to the spatially averaged species mass fractions of incoming field for the DNS. Such laminar flames are called MILD Flame Element (MIFE) to differentiate them from standard flamelets and Table 1 also summarises the thermochemical conditions of these MIFEs. The flame speed and thickness of these MIFEs are used to normalise DNS quantities for MILD combustion in this study.

All of the present DNS conditions have an equivalence ratio of $\phi=0.8$. Specifically, for the MILD combustion $\phi_{i n j}$ marked in Fig. 2 is 0.8 and the autoiginition temperature is $1100 \mathrm{~K}$. The inlet and initial mixture temperatures are 
Table 1: Thermochemical conditions of 1D canonical laminar flames.

\begin{tabular}{cccccccccc}
\hline Flame & $X_{\mathrm{CH}_{4}, r}$ & $X_{\mathrm{O}_{2}, r}$ & $X_{\mathrm{H}_{2} \mathrm{O}, r}$ & $X_{\mathrm{CO}_{2}, r}$ & $T_{r}(\mathrm{~K})$ & $T_{p}(\mathrm{~K})$ & $\tau_{i g n}(\mathrm{~ms})$ & $S_{L}(\mathrm{~m} / \mathrm{s})$ & $\delta_{F}(\mathrm{~mm})$ \\
\hline Flame A & 0.019 & 0.048 & 0.121 & 0.061 & 1500 & 1865 & 4.97 & 3.20 & 0.116 \\
Flame B & 0.014 & 0.035 & 0.132 & 0.066 & 1500 & 1775 & 6.71 & 2.15 & 0.168 \\
Flame C & 0.078 & 0.194 & 0.0 & 0.0 & 600 & 2179 & - & 1.18 & 0.064 \\
& & & & & & & & & \\
MIFE A & 0.0095 & 0.035 & 0.136 & 0.064 & 1500 & 1692 & 5.42 & 2.62 & 0.138 \\
MIFE B & 0.006 & 0.025 & 0.143 & 0.068 & 1500 & 1624 & 6.96 & 1.66 & 0.217 \\
\hline
\end{tabular}

set to be $T_{m} \approx 1500 \mathrm{~K}$ for the MILD combustion cases, which is comparable to that used in [39]. This inlet temperature together with the dilution level used in this study (see Tables 1 and 2) shows that the combustion is strictly in the MILD regime as shown in Fig. 3a for the conditions of the Flames A and B. A small temperature rise across the MILD combustion zone, compared to a premixed flame, is shown in Fig. 3b. The temperature rise across the reaction zones of the Flames $\mathrm{A}$ and $\mathrm{B}$ is larger than that for the respective MIFEs. The reactant temperature for the conventional turbulent premixed flame, Case $\mathrm{C}$ is set to be $600 \mathrm{~K}$ as noted in Table 1. The mixture and turbulence conditions of the three turbulent MILD combustion cases considered in this study are given in Table 2. The maximum and averaged molar fractions of oxygen in the reactant mixtures for the MILD cases are nearly an order of magnitude smaller than for the premixed case, Case C. The normalised mean inflow velocity, $U_{i n} / S_{L}$, is 9.6, 15.1, and 3.0 for the Cases A, B and C respectively. The r.m.s. of velocity fluctuations and the integral length scale of the initial turbulence field are denoted respectively as $u^{\prime}$ and $l_{0}$ in Table 2. Although the turbulence Reynolds number $R e_{l_{0}}$ is small, it is representative of typical values observed in experiments $[40,41,42]$ and in furnaces [21, 43, 7] with MILD combustion conditions. The Damköhler and Karlovitz 
Table 2: Three-dimensional DNS conditions for the MILD and conventional combustion.

\begin{tabular}{cccccccccc}
\hline Case & $X_{\mathrm{O}_{2}, r}^{\max }$ & $\left\langle X_{\mathrm{O}_{2}, r}\right\rangle$ & $U_{i n} / S_{L}$ & $u^{\prime} / S_{L}$ & $l_{0} / \delta_{F}$ & $l_{0} / \delta_{t h}$ & $\mathrm{Re}_{l_{0}}$ & $\mathrm{Da}$ & $\mathrm{Ka}$ \\
\hline $\mathrm{A}$ & 0.048 & 0.035 & 9.6 & 3.80 & 12.3 & 1.70 & 67.0 & 3.25 & 2.11 \\
$\mathrm{~B}$ & 0.035 & 0.025 & 15.1 & 9.88 & 6.8 & 1.15 & 96.1 & 0.69 & 11.9 \\
C & 0.194 & 0.194 & 3.0 & 2.19 & 12.3 & 2.11 & 38.5 & 5.64 & 0.92 \\
\hline
\end{tabular}

numbers are defined as $\mathrm{Da}=\left(l_{0} / \delta_{F}\right) /\left(u^{\prime} / S_{L}\right)$ and $\mathrm{Ka} \approx\left(u^{\prime} / S_{L}\right)^{3 / 2}\left(l_{0} / \delta_{F}\right)^{-1 / 2}$ respectively. The thermal thickness of the respective one-dimensional unstrained laminar flame is defined as $\delta_{t h}=\left(T_{p}-T_{r}\right) /|\nabla T|_{\max }$. The first two cases, A and $\mathrm{B}$, are in the thin-reaction zones regime and Case $\mathrm{C}$ is near the border between the thin-reaction zones and corrugated flamelets regimes in the classical turbulent combustion regime diagram [44].

To make the conditions of the three flames clearer, there is one premixed, Case C, and two MILD, Cases A and B, combustion cases. The premixed case is used for comparative analysis and to contrast the behaviour of MILD reaction zones. The Cases A and B are chosen carefully so that they are representative of experimental MILD cases, as noted above, and yet have an order of magnitude difference in Da values. This is achieved by careful selection of turbulence and thermo-chemical conditions noted in Tables 1 and 2. The Da and Ka values are kept similar for the Cases $\mathrm{A}$ and $\mathrm{C}$ to see if the dilution affects the reaction zone characteristics. Indeed, as one shall see in later sections of this paper the Case A shows distinctive changes in the reaction zone structure and behaviour.

The computational domain has dimensions of $L_{x} \times L_{y} \times L_{z}=10.0 \times 10.0 \times$ $10.0 \mathrm{~mm}^{3}$ for the Cases $\mathrm{A}$ and $\mathrm{B}$, and $L_{x} \times L_{y} \times L_{z}=10.0 \times 5.0 \times 5.0 \mathrm{~mm}^{3}$ for the Case $\mathrm{C}$. These domain sizes give about 4 to 7 integral length, $l_{0}$, in each 
direction. These domains are discretised using $512 \times 512 \times 512$ mesh points for the Case A, $384 \times 384 \times 384$ mesh points for the Case B, and $512 \times 256 \times 256$ mesh points for the Case $\mathrm{C}$. These meshes ensure that there are at least 15 mesh points inside $\delta_{t h}$ to resolve chemical structure as has been shown in many earlier studies, see for example [45].

The simulations of the MILD cases, A and B, were run for 1.5 flow-through times before collecting data for statistical analysis. This ensured that the initial transients had left the domain. The flow-through time $\tau_{D}$ is defined as the mean convection time, $L_{x} / U_{i n}$, from the inflow to the outflow boundary. The simulations were then continued for one additional flow-through time and 80 data sets (all the primitive variables) were collected. For the Case C, 93 data sets were collected over a time of $0.56 \tau_{D}$ after allowing one flow-through time for initial transients to exit the computational domain. These simulations have been run on Cray XE6 systems using 4096 cores with a wall-clock time of about 120 hours for the Case A, which has the largest number of mesh points among the MILD cases, and using 16384 cores with 80 hours of wall-clock time for the Case C.

\section{Reaction zone and progress variable variations}

Figure 4 shows contours of the reaction rate field $\omega_{c_{T}}^{*}$ in the mid $x-y$ plane at $t=1.5 \tau_{D}$ for the three cases considered. The reaction rate is obtained as $\omega_{c_{T}}=\dot{Q} / c_{p}\left(T_{p}-T_{r}\right)$, where $\dot{Q}$ and $c_{p}$ denote respectively the heat release rate and the specific heat capacity of the local mixture. The superscript “*” denotes a normalisation using the global maximum value in the corresponding two-dimensional slice. The quantities with superscript "+" denote values appropriately normalised using the reactant density $\rho_{r}$ and the respective laminar flame (MIFEs for the 


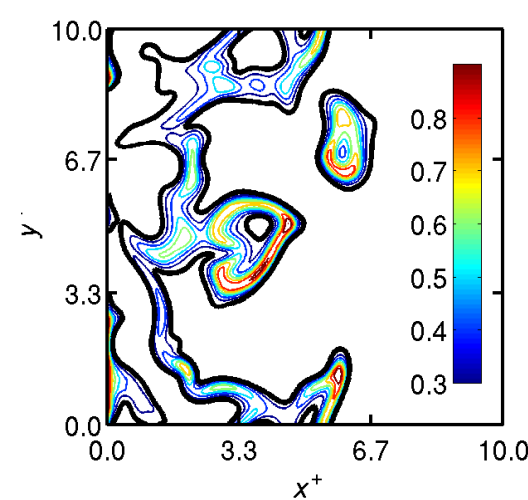

(a)

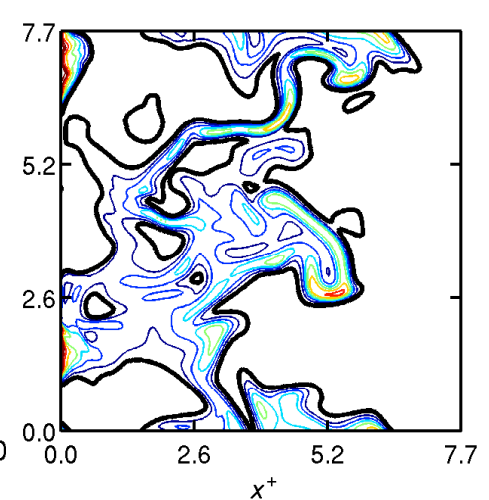

(b)

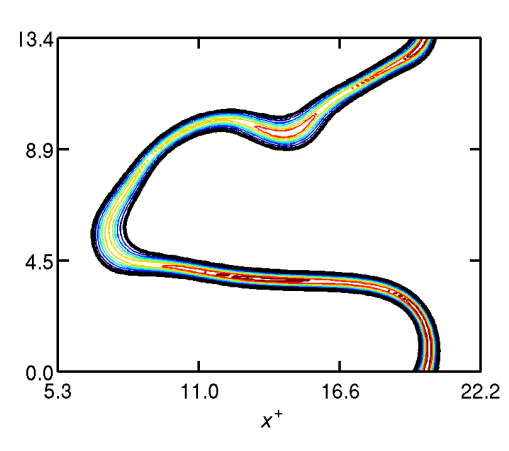

(c)

Figure 4: (Colour online) Contours of $\omega_{c_{T}}^{*}$ in the mid $x-y$ plane at $t=1.5 \tau_{D}$ for (a) Case A, (b) Case B and (c) Case C. Thick black line:0.2, and thin lines: $0.3,0.4, \cdots, 0.9$ as indicated by the colour-map. The axes are normalised using $\delta_{t h}$.

MILD cases) quantities, $S_{L}$ and $\delta_{t h}$. For example, length, gradient of reaction progress variable and reaction rate are respectively normalised using $\delta_{t h}, 1 / \delta_{t h}$ and $\rho_{r} S_{L} / \delta_{t h}$. The superscript “**” appearing later in this paper denotes a normalisation using the global maximum value in the entire 3D domain.

The normalised reaction rate shown in Figs. $4 \mathrm{a}$ and $4 \mathrm{~b}$ respectively for Cases $\mathrm{A}$ and $\mathrm{B}$, suggests that intense chemical activities appear in locally thin reaction zones. These regions are highly convoluted compared to the conventional premixed case shown in Fig. 4c, and the thin reaction zones in the premixed combustion case has a nearly constant thickness of about $\delta_{t h}$. The degree of convolution increases with the levels of turbulence and dilution (compare Figs. $4 \mathrm{a}$ and $4 \mathrm{~b}$ ). A comparison between Cases A (Fig. 4a) and C (Fig. 4c) shows that the level of convolution is large under the MILD combustion condition. In general, the degree of flame wrinkling in turbulent premixed combustion strongly depends on the turbu- 
lence level. The spatial non-uniformity of the mixture for the MILD combustion also affects the flame wrinkling, since the local reaction rate is influenced by the mixture non-uniformity. This enhanced wrinkling leads to interaction of reaction zones as has been observed in [8].

As described in the introduction, the reaction rate variation is estimated adequately using PLIF signals of specific species such as $\mathrm{OH}, \mathrm{CH}_{2} \mathrm{O}$ and $\mathrm{CHO}$ for conventional premixed turbulent combustion in the past studies $[11,12,13,15]$. However these methods have not been validated for MILD combustion yet. The PLIF signal of a species $i, S_{i}$, is related to the local species molar concentration and temperature. This signal can be represented by:

$$
\begin{array}{r}
S_{\mathrm{CH}_{2} \mathrm{O}} \propto\left[\mathrm{CH}_{2} \mathrm{O}\right] T^{1-\beta}, 2.2 \leq \beta \leq 3.0 \\
S_{\mathrm{OH}} \propto[\mathrm{OH}] T^{1-\beta}, \quad-2.0 \leq \beta \leq 1.0 \\
S_{\mathrm{CHO}} \propto[\mathrm{CHO}] T^{1-\beta}, \quad 1.0 \leq \beta \leq 1.5,
\end{array}
$$

for a temperature range of $1000 \leq T \leq 1800 \mathrm{~K}$ when Boltzmann equilibrium is considered [11, 19, 46, 47, 48]. Here, the square brackets denotes molar concentration of species, and the parameter $\beta$ can be adjusted by the selection of particular transition used for the measurement. For the present study, the parameter $\beta$ is set to be 2.6 for $\mathrm{CH}_{2} \mathrm{O}$, zero for $\mathrm{OH}$ and 1.25 for $\mathrm{CHO}$ signals $[11,19]$. It is also verified that the choice of $\beta$ does not change the conclusions of the present study by eliminating the temperature dependence in Eqs. (1)-(3) by setting $\beta=1$.

Figure 5 shows variations of the actual reaction rate $\omega_{c_{T}}^{*}$, and the estimated reaction rates across the laminar flames discussed in Fig. 3b. The estimated reaction rates are $\omega_{e 1}^{*}=\left(S_{\mathrm{OH}} \times S_{\mathrm{CH}_{2} \mathrm{O}}\right)^{*}$ and $\omega_{e 2}^{*}=S_{\mathrm{CHO}}^{*}$. The peak locations of the actual 


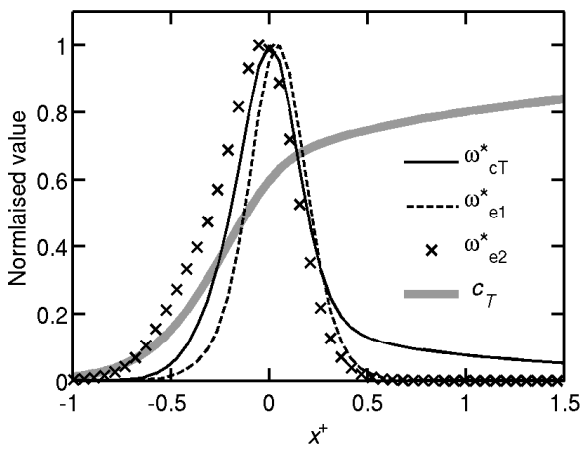

(a)

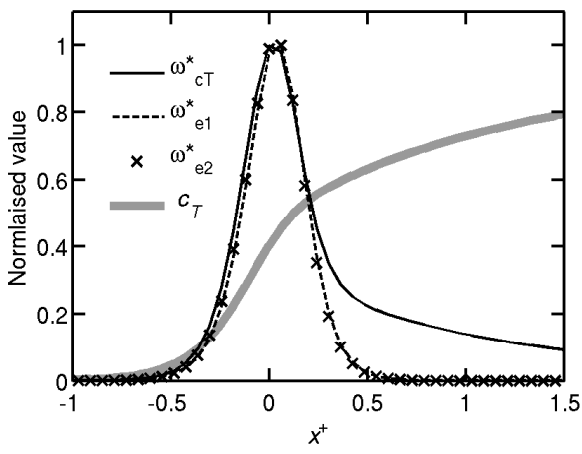

(b)

Figure 5: Variations of the actual ( $\omega_{c_{T}}^{*}$ : solid black line), estimated ( $\omega_{e 1}^{*}$ : dashed line, and $\omega_{e 2}^{*}: \times$ ) reaction rates, and $c_{T}$ (thick grey line) for the respective laminar flames for (a) Case C (Flame C) and (b) Case B (MIFE B). The location $x^{+}=0$ corresponds to the location of peak $\omega_{c_{T}}^{*}$, and $x^{+}<0$ and $x^{+}>0$ correspond to unburnt and burnt sides respectively.

and estimated reaction rates are almost identical with a small difference of about $0.06 \delta_{t h}$ for the MILD laminar flame, and about $0.11 \delta_{t h}$ for the premixed laminar flame. However, the variation of the actual reaction rate across the flame is reproduced only approximately by the estimates for premixed flames, as reported in [13]. Specifically, the non-zero reaction rate on the burnt side is captured by neither of the estimates, since both $\mathrm{CH}_{2} \mathrm{O}$ and $\mathrm{CHO}$ are consumed quickly in the reaction zone. The estimate $\omega_{e 1}^{*}$ is reasonable and can be used to identify heat releasing zones as has been done in previous experimental studies using PLIF techniques for premixed combustion $[11,12,13,16,49,17,18]$. However, these LIF markers have not been verified for MILD mixtures, thus it is worthwhile to test these markers for turbulent MILD cases. This is because the reactant mixture is non-uniform, which would influence reaction zone shape and its location. Figure $5 \mathrm{~b}$ compares the actual and estimated reaction rates for MIFE B. Except for 
the burnt side, the comparison shows good agreement.

The typical estimated reaction rate fields $\omega_{e 1}^{*}$ and $\omega_{e 2}^{*}$ in the same $x-y$ plane as in Fig. 4 are respectively shown in Figs. 6(a-c) and (d-f). The results are shown for both MILD and premixed cases. These estimated fields show reasonable agreement with the actual field shown in Fig. 4. It is not surprising to see good agreement for the premixed case. Many features of the MILD reaction zones observed in Fig. 4 are reproduced reasonably well in Fig. 6. There are some differences also; for example, the shape of isolated pockets of reaction zones in the estimated field for the Case B (high dilution case) in Figs. 6b and 6e. However, the location of local maximum reaction rate is well represented in the estimated fields. Such observation has been made for premixed flames in earlier studies [11, 20, 13].

Figure 7 shows the variations of instantaneous $\omega_{e 1}^{*}$ and $\omega_{e 2}^{*}$ with $\omega_{c_{T}}^{*}$ as a scatter plot for the Case B. The samples are obtained from the $x-y$ plane shown in Fig. 6 , and this variation is found to be typical for other MILD and premixed cases. The correlation between the actual and estimated values is relatively low for small values of $\omega_{c_{T}}^{*}$ with two distinct branches as shown in Fig. 7. The estimated reaction rate across the flame agrees well with the actual variation in unburnt side as in Fig. 5b, which corresponds to the left branch in Fig. 7, while the estimated value tends to be smaller than the actual value in burnt side (the right branch). However, the agreement of the actual and estimated values is good for high reaction rate $\left(\omega_{c_{T}}^{*} \geq 0.3\right)$, and thus the estimation of the reaction rate field using the chemical markers $S_{\mathrm{OH}} \times S_{\mathrm{CH}_{2} \mathrm{O}}$ and $S_{\mathrm{CHO}}$ is reasonable for the MILD combustion as well as the conventional premixed combustion.

The gradient of the progress variable is of interest in this study as noted in the Introduction. Thus, the contours of reaction progress variable based on temper- 


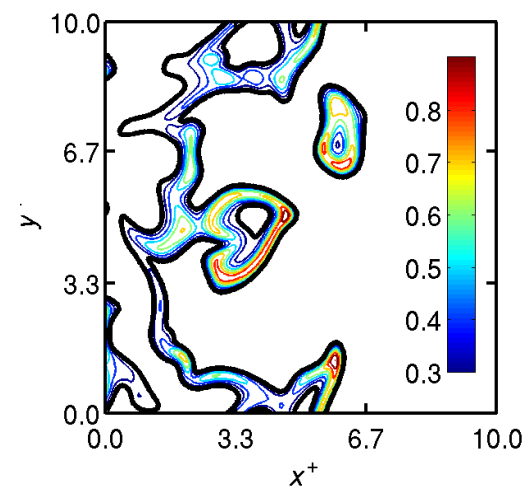

(a)

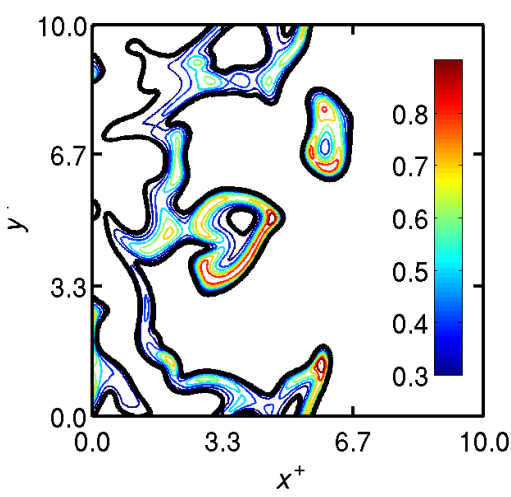

(d)

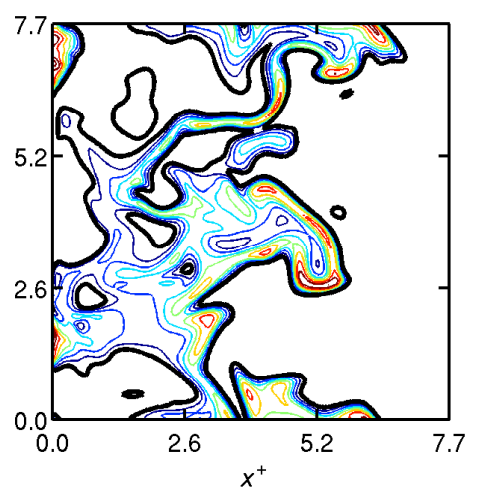

(b)

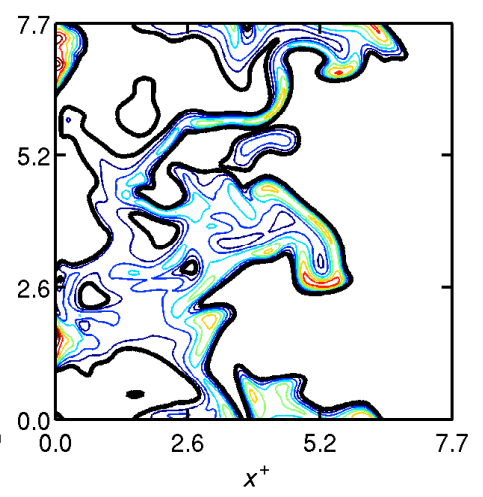

(e)

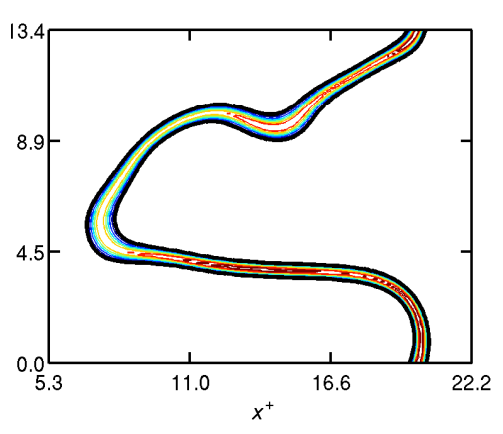

(c)

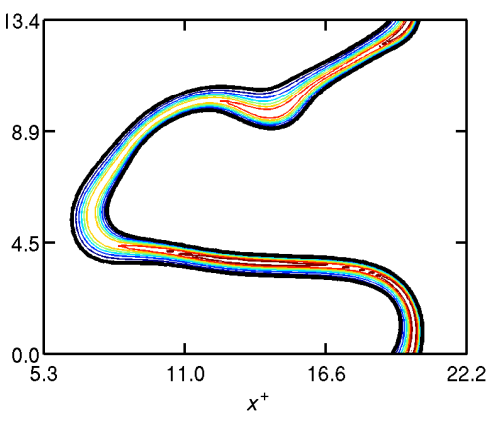

(f)

Figure 6: (Colour online) Contours of $\omega_{e 1}^{*}(\mathrm{a}-\mathrm{c})$, and $\omega_{e 2}^{*}(\mathrm{~d}-\mathrm{f})$ in the same $x-y$ plane as Fig. 4 for Case A (a, d), Case B (b, e) and Case C (c, f). Thick black line: 0.2 , and thin lines: $0.3,0.4, \cdots, 0.9$ as indicated by the colour-map. 


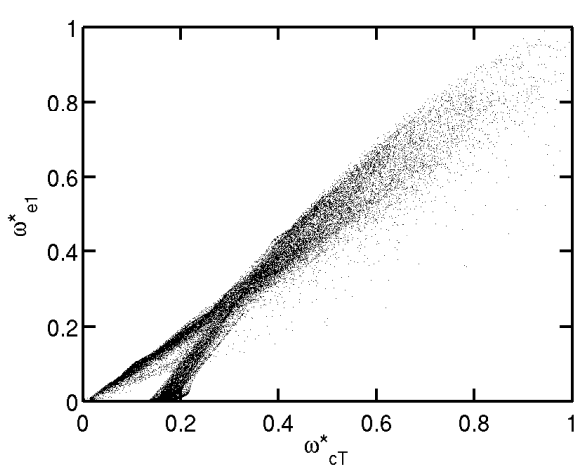

(a)

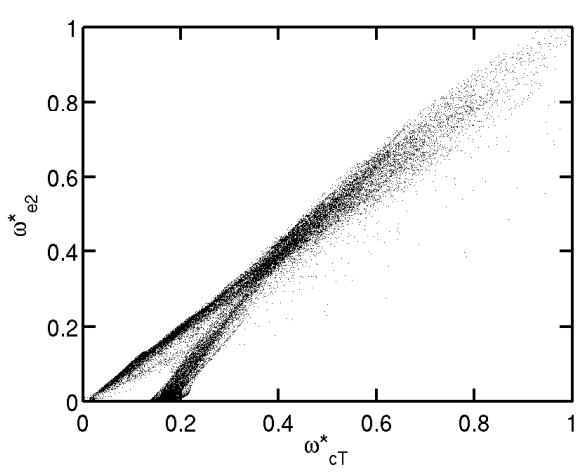

(b)

Figure 7: Typical variations of instantaneous $\omega_{e 1}^{*}$ and $\omega_{e 2}^{*}$ with $\omega_{c_{T}}^{*}$ for Case B. One fourth of all the samples from the same $x-y$ slice as in Fig. 6 are shown.

ature, $c_{T}=\left(T-T_{r}\right) /\left(T_{p}-T_{r}\right)$, are compared to those constructed using $S_{\mathrm{OH}}^{*}$. The typical results are shown in Fig. 8 for the Cases B and C. The $c_{T}$ contours are shown as solid lines while the contours of the estimated progress variable field $c_{\mathrm{PLIF}}$ are shown as dashed lines. The $c_{\mathrm{PLIF}}$ contours are obtained as $c_{\mathrm{PLIF}}=S_{\mathrm{OH}}^{*}$, where $S_{\mathrm{OH}}^{*}$ varies from zero to unity. This quantity $S_{\mathrm{OH}}^{*}$ is similar to that could be obtained in OH PLIF measurements. As one would expect, the $c_{\text {PLIF }}$ field reproduces the characteristics of $c_{T}$ well for the premixed case, except for a small offset. The scatters of instantaneous $c_{T}$ and $c_{\text {PLIF }}$ shown in Fig. 9a also suggests that $c_{T}$ can be reconstructed once local $c_{\mathrm{PLIF}}$ is known, since the relationship between instantaneous $c_{T}$ and $c_{\mathrm{PLIF}}$ is monotonic except for $c_{\mathrm{PLIF}} \geq 0.85$. This further confirms that the statistics of $c_{T}$ and $c_{\mathrm{PLIF}}$ is similar to one another for the premixed case.

A comparison of $c_{T}$ and $c_{\text {PLIF }}$ shown for MILD combustion, Case B, in Figs. 8d to $8 \mathrm{f}$ does not show as good correlation as for the premixed case in general. However, a good agreement is observed for $c_{T}=0.6$ and $c_{\mathrm{PLIF}}=0.6$ contours, where 


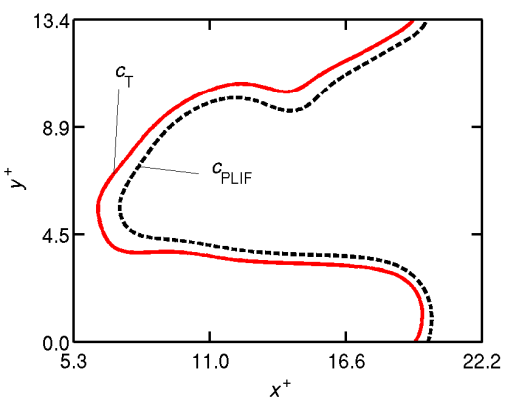

(a)

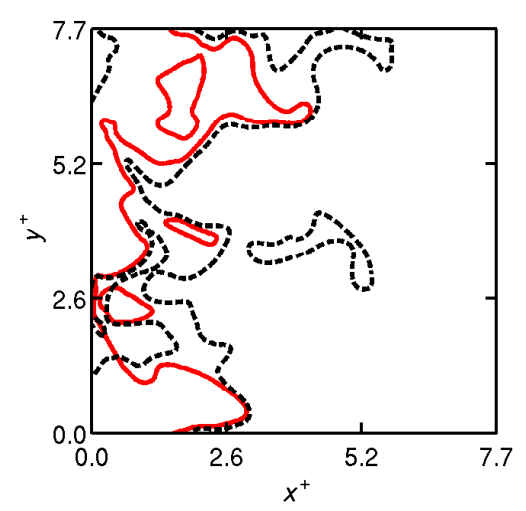

(d)

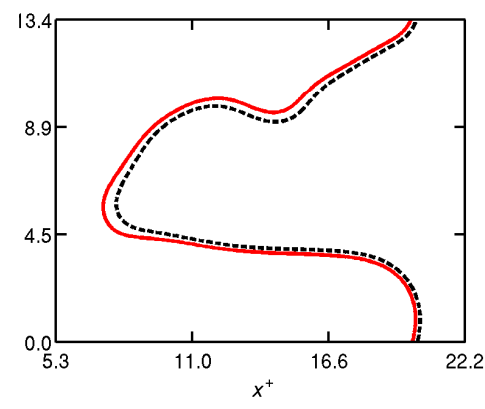

(b)

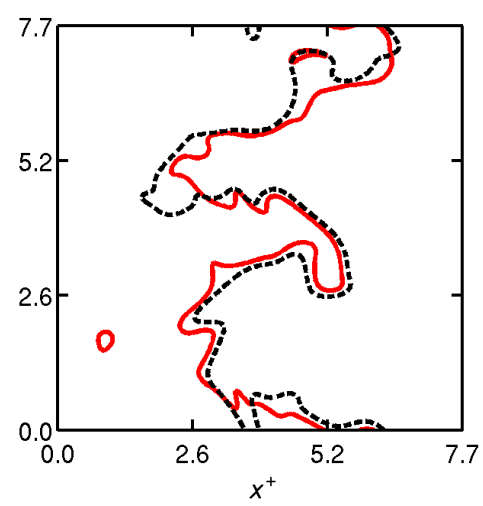

(e)

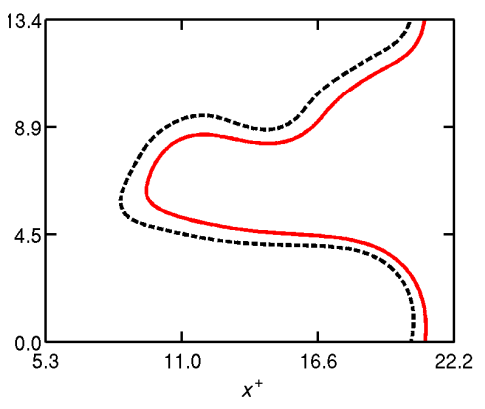

(c)

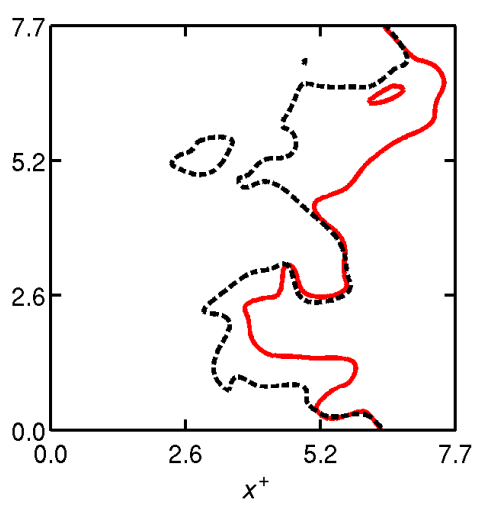

(f)

Figure 8: (Colour online) Contours of $c_{T}$ (solid-red line) and $c_{\text {PLIF }}$ (dashed line) for Case $\mathrm{C}(\mathrm{a}-\mathrm{c})$ and Case B (d-f) in the same $x-y$ plane as in Fig. 4. The contour level is $0.2(\mathrm{a}, \mathrm{d}), 0.6(\mathrm{~b}, \mathrm{e})$ and $0.8(\mathrm{c}, \mathrm{f})$. 


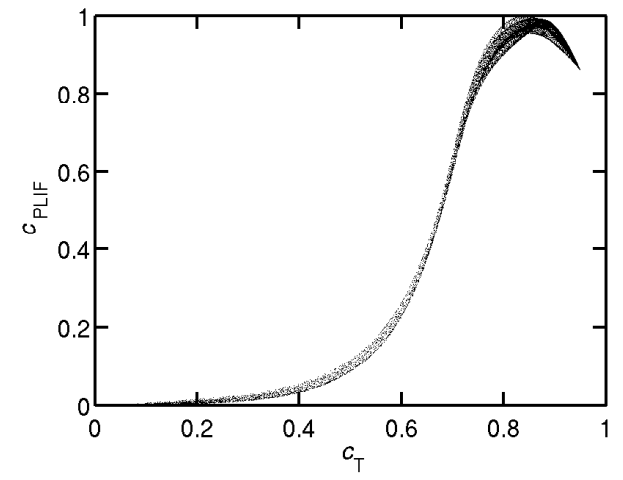

(a)

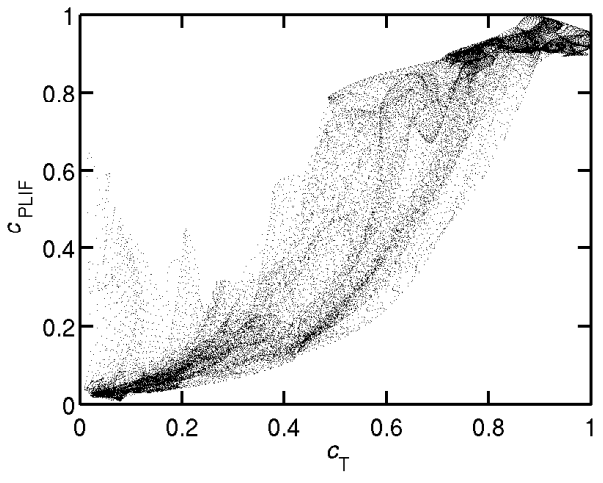

(b)

Figure 9: Scatter plot of the instantaneous $c_{T}$ and $c_{\text {PLIF }}$ for (a) Case C and (b) Case B. One fourth of all the samples in the same $x-y$ slice as in Fig. 6 are shown above.

the heat release peaks in the respective canonical laminar flame. For other $c_{T}$ contours shown in Figs. 8d and 8f, there is a close match observed locally, between $c_{T}$ and $c_{\text {PLIF }}$ in several locations. Generally the representation of $c_{T}$ by $c_{\text {PLIF }}$ in MILD combustion is not as good as for the premixed case. The broad distribution of the $c_{T}-c_{\text {PLIF }}$ scatter in Fig. 9b also suggests this.

A close study of Fig. 8 suggests that the $c_{T}$ and $c_{\text {PLIF }}$ contours are almost parallel to one another in predominant portions for the MILD combustion case. Thus a contour of particular $c_{T}$ value can be represented well by a contour of different $c_{\text {PLIF }}$ value. This parallelism suggests that their gradient vectors point in the same direction, which is verified by calculating the PDF of the inner product of the gradient unit vectors conditioned on $\omega_{c_{T}}^{*}$. These unit vectors are defined as $\boldsymbol{n}_{c_{T}}=\nabla c_{T} /\left|\nabla c_{T}\right|$ and $\boldsymbol{n}_{\mathrm{OH}}=\nabla c_{\mathrm{PLIF}} /\left|\nabla c_{\mathrm{PLIF}}\right|$. The typical PDF of the inner product conditioned on the reaction rate, $\omega_{c_{T}}^{*}$ calculated as $P\left(\left|\boldsymbol{n}_{\mathrm{OH}} \cdot \boldsymbol{n}_{c_{T}}\right| \mid \omega_{c_{T}}\right)=$ $P\left(\left|\boldsymbol{n}_{\mathrm{OH}} \cdot \boldsymbol{n}_{c_{T}}\right|, \omega_{c_{T}}\right) / P\left(\omega_{c_{T}}\right)$, is shown in Fig. 10 for the MILD (Case B) and pre- 


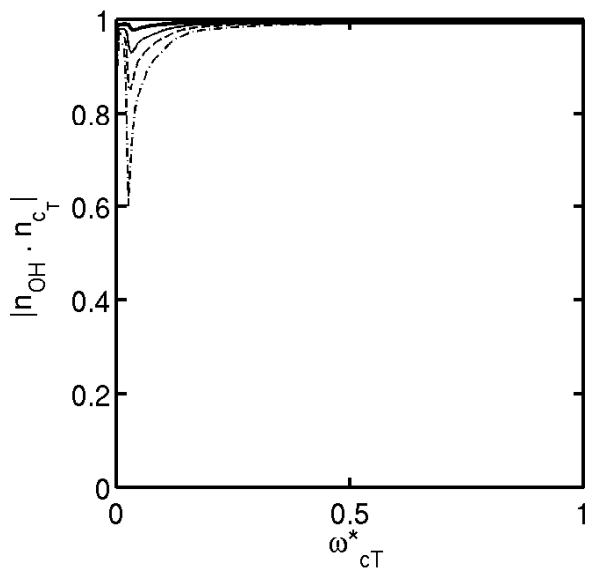

(a)

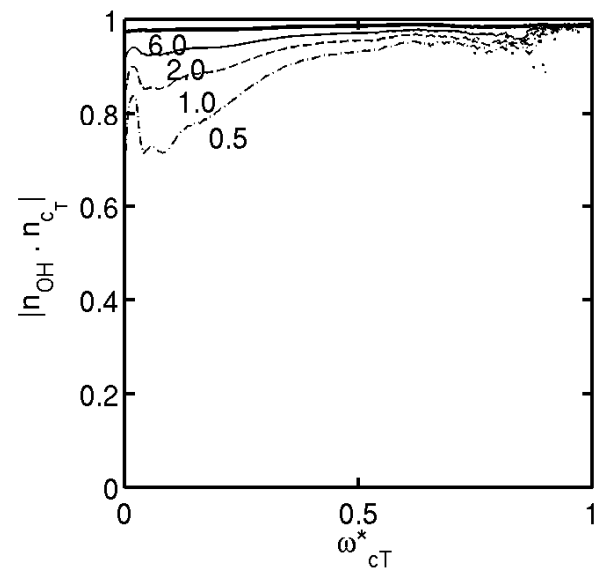

(b)

Figure 10: Contours of the conditional PDF of the inner product, $p\left(\mid \boldsymbol{n}_{c_{T}}\right.$. $\left.\boldsymbol{n}_{\mathrm{OH}}|| \omega_{c_{T}}^{*}\right)$ for (a) Case C and (b) Case B. The PDF is constructed using samples from the entire domain and sampling period for each case.

mixed (Case C) cases. Here, $P\left(\omega_{c_{T}}^{*}\right)$ is the marginal PDF of $\omega_{c_{T}}^{*}$. The conditional PDF shown for the premixed case peaks near $\left|\boldsymbol{n}_{c_{T}} \cdot \boldsymbol{n}_{\mathrm{OH}}\right|=1$ as one would expect. The PDF for the Case B shown in Fig. 10b is typical for the MILD combustion cases studied here. The PDF shown for the MILD case also suggests a good alignment between $\boldsymbol{n}_{c_{T}}$ and $\boldsymbol{n}_{\mathrm{OH}}$, specifically at locations with high heat release rate. The PDF broadens slightly for small $\omega_{c_{T}}^{*}$ values, which come predominantly from unburnt and burnt regions of the flame front, as shown in Fig. 5b. It has been observed in Figs. 8d and $8 \mathrm{f}$ that the $c_{T}$ and $c_{\mathrm{PLIF}}$ contours are less likely to be parallel at regions with $c_{T}$ close to zero and unity. The tendency for the alignment of these two gradients is high for $\omega_{c_{T}}^{*} \geq 0.3$. Therefore, the orientation of the $c_{T}$ gradient can be estimated adequately using variation of $S_{\mathrm{OH}}^{*}$ gradient to deduce further insight on the scalar gradient behaviour. Also, the reaction rate signal, $\omega_{e 1}^{*}$, can be obtained relatively easily using $S_{\mathrm{OH}}$ and $S_{\mathrm{CH}_{2} \mathrm{O}}$. Thus, the reaction rate can 
be used to condition the scalar gradient PDF as has been done in the next section.

\section{Scalar gradient behaviour}

Strong scalar gradient due to the intense chemical activity within thin regions characterises flamelet like combustion. Also, the flame normal component of the scalar gradient is statistically very large compared to the tangential component in the flamelet combustion. Hence the relationship between the normal component of the scalar gradient and reaction rate in turbulent premixed flame would be similar to that of the respective laminar flame solution. The characteristics of this relationship can be understood by investigating the PDF of the scalar gradient conditioned on the reaction rate, which is discussed in this section. First, a method to construct this PDF from the DNS data is described. Then, this PDFs obtained using the DNS and synthesised LIF fields are compared to assess the validity of the statistics deduced from the synthesised fields. This approach is also applied to the 2D field constructed from the DNS since 2D measurements are quite common in laser diagnostics investigation. The comparison of 2D and 3D results (for both DNS and constructed LIF fields) will help us to see if the laboratory measurements can be used to construct these conditional PDFs.

\subsection{Data analysis}

The method used here to construct the conditional PDF from the complete three dimensional fields of reaction rate and scalar gradient involves five steps given below. A quantity normalised using its maximum value in the entire domain is denoted using a superscript "**" in the following discussion.

1. The three dimensional gradient of progress variable $\nabla c$, either $c_{T}$ or $c_{\text {PLIF }}$, 
is computed from the primitive variables using the same numerical scheme employed for the DNS.

2. The flame surface is identified using the local minimum value of $\nabla \omega^{* *}$ subject to $\omega^{* *}>0.5$. The method identifies the local maximum reaction rate which correlates well with the estimates obtained using the deduced LIF signals as shown in Fig. 5. Furthermore, it helps to avoid multiply connected surfaces, which would result if a threshold value is used, especially in the MILD cases (see Fig. 4). The surface identified thus is expressed as $\left(f_{x}(s), f_{y}(s)\right)$ for $x-y$, and $\left(f_{x}(s), f_{z}(s)\right)$ for $x-z$ planes, where $s$ is a local coordinate along the surface.

3. The normal vectors, $\boldsymbol{n}_{1, x y}$ and $\boldsymbol{n}_{1, x z}$, of the surfaces are calculated in every 2D plane as $\boldsymbol{n}_{1, x y}=\left(-d f_{y} / d s, d f_{x} / d s\right)$ for $x-y$ and $\boldsymbol{n}_{1, x z}=\left(-d f_{z} / d s, d f_{x} / d s\right)$ for $x-z$ planes. Similar procedure is followed to obtain the tangential vectors, $\boldsymbol{n}_{2, x y}$ and $\boldsymbol{n}_{2, x z}$, in the corresponding planes.

4. The flame normal and one of its two tangential vectors at every point on the surface is then constructed using the respective $2 \mathrm{D}$ vectors obtained in the previous step. The second tangential vector is calculated using the vector cross product rule. The normal and tangential components of $\nabla c$ are then computed as $\nabla c \cdot \boldsymbol{n}_{\boldsymbol{i}}$ for $c_{T}$ and $c_{\mathrm{PLIF}}$, where $i=1,2$ and 3 denote respectively the normal and two tangential directions.

5. The samples for constructing the PDF are collected in the normal and tangential directions over a length of $2 \delta_{t h}$ on either side of the point of interest. These samples obtained from the entire computational domain are used to 
construct joint, $P\left(\psi_{i}^{+}, \omega\right)$, and marginal, $P(\omega)$, PDFs. The symbol $\psi_{i}^{+}$denotes $\ln \left(\left|\nabla^{+} c \cdot \boldsymbol{n}_{i}\right|\right)$ and $\omega$ denotes $\omega_{c_{T}}^{+}$or $\omega_{e 1}^{* *}$ or $\omega_{e 2}^{* *}$. The conditional PDF is then obtained using the Bayes theorem: $P\left(\psi_{i}^{+} \mid \omega\right)=P\left(\psi_{i}^{+}, \omega\right) / P(\omega)$.

The above steps are repeated to get the conditional PDF from 2D subsets of the 3D data. The only difference is in the steps 3 and 4 described above for the normal and tangential vector calculation. These vectors are obtained simply as $\boldsymbol{n}_{1}=\left(-d f_{y} / d s, d f_{x} / d s\right)$ and $\boldsymbol{n}_{2}=\left(d f_{x} / d s, d f_{y} / d s\right)$ in a given 2D $(x-y)$ plane.

\subsection{Conditional PDFs of scalar gradients}

Contours of the conditional PDF, $P\left(\psi_{i}^{+} \mid \omega^{* *}\right)$ of 3D scalar gradient constructed using $c_{T}$ and $\omega_{c_{T}}^{+}$, and the deduced LIF signals are shown in Fig. 11 for the two MILD and premixed cases. The premixed case is shown in the first column and the MILD cases are shown in the second and third columns. The PDFs constructed using $c_{T}$ and $\omega_{c_{T}}^{+}$are in the top row and the other two rows show the PDFs for the synthesised LIF signals. The conditional PDF for the normal component is shown using solid lines and the tangential component is shown using the dashed line. Since the conditional PDFs of the tangential components $\psi_{2}^{+}$and $\psi_{3}^{+}$are almost identical, the conditional PDF is shown only for $\psi_{2}^{+}$. The grey line denote the respective laminar flame (MIFEs for the MILD cases) solution $\left(\psi_{\text {lam }}^{+}, \omega_{c_{T}}^{* *}\right)$. The laminar curves shown in the bottom two rows of Fig. 11 are obtained using the LIF signals deduced from the respective laminar flame solution as explained in section 3 .

The conditional PDF for the premixed case in Fig. 11a shows that the flame normal component is larger than the tangential component when the reaction rate is large $\left(\omega_{c_{T}}^{* *}>0.5\right)$. The high probability density for the normal component exists in relatively narrow region compared to the tangential component. This region 
is well bounded by the laminar flame solution suggesting that the probability of finding high gradient similar to that in the laminar flame is large. For tangential component, the PDF is distributed over a wider range of $\omega_{c_{T}}^{* *}$ and $\psi^{+}$and the peak PDF occurs for low reaction rate and scalar gradient values. The probability of finding high value of tangential gradient in regions of high reaction rate is negligibly small. This is what one would see in turbulent premixed combustion in flamelets regime and the PDFs from this case serves as a baseline behaviour to compare with. The conditional PDFs obtained using the synthesised two LIF signals, one using $S_{\mathrm{OH}}$ and $S_{\mathrm{CH}_{2} \mathrm{O}}$, and another one using $S_{\mathrm{CHO}}$, as explained in section 3 are similar to that for the DNS data (cf. Figs. 11a, 11d and 11g). The corresponding laminar solutions shown in Fig. 11d and $11 \mathrm{~g}$ also suggests the flamelet combustion. These laminar solutions show two values of the normalised scalar gradient for a given value of the normalised reaction rate. This is because of some difference in the location of peak values of the reaction rate and scalar gradient in the laminar flame. The larger values of the scalar gradient come from the reaction zone and the lower values are from either the preheat or hotter part of the reactive-diffusive regions. The gap between these two branches is the largest when $S_{\mathrm{CHO}}$ is used in the analysis. However, the relative behaviour between the turbulent case and the respective flamelet is maintained clearly showing the expected flamelet behaviour.

The conditional PDFs for the MILD cases show distinct changes. The PDFs for the normal and tangential components are very close for low reaction rate values unlike for the premixed case. The PDF contours for the tangential and normal components start to deviate slowly from each other as the reaction rate increases. This deviation starts to occur at about $\omega_{c_{T}}^{* *} \approx 0.2$ in the Case A and 


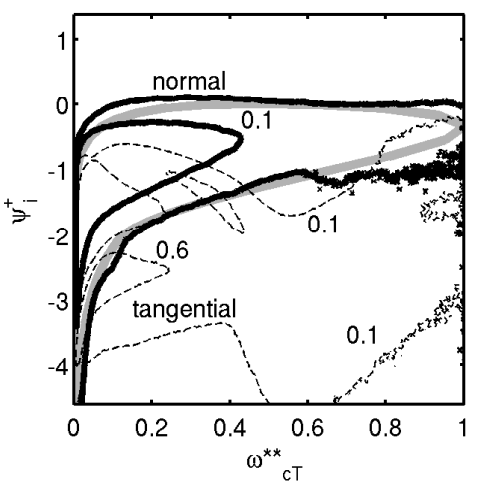

(a)

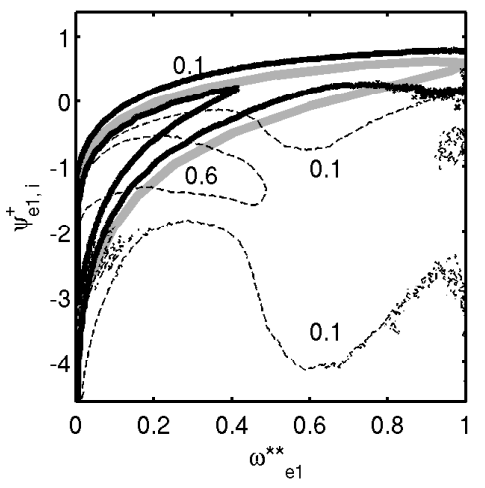

(d)

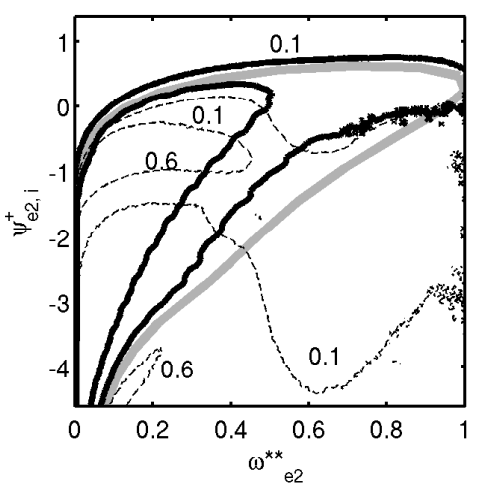

(g)

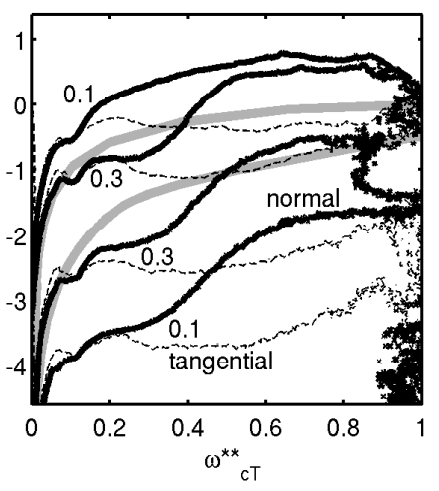

(b)

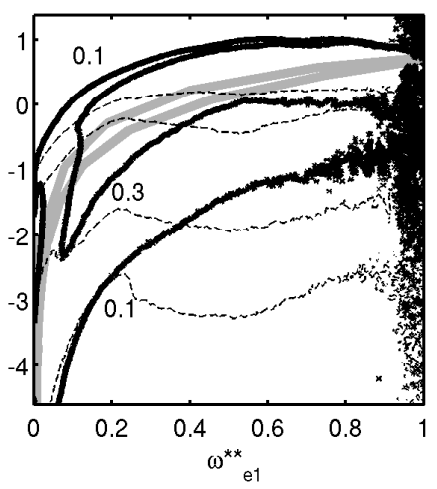

(e)

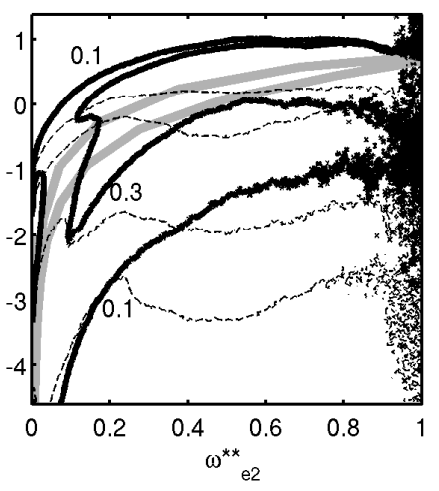

(h)

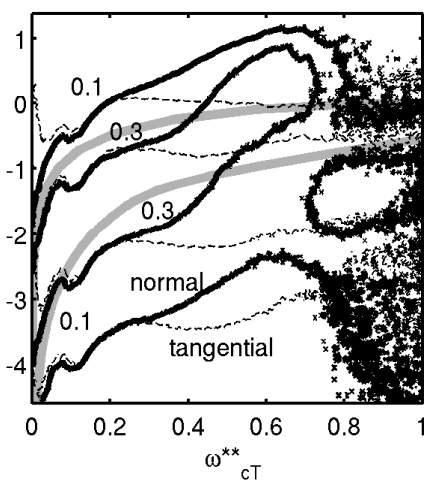

(c)

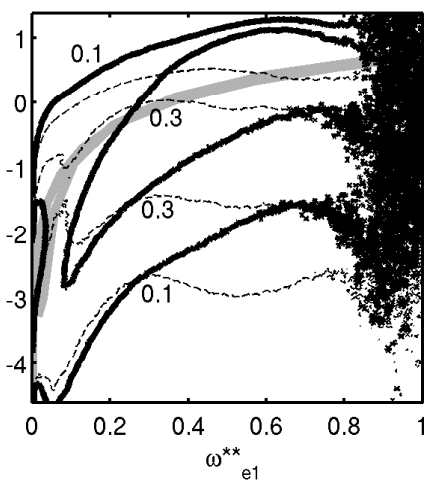

(f)

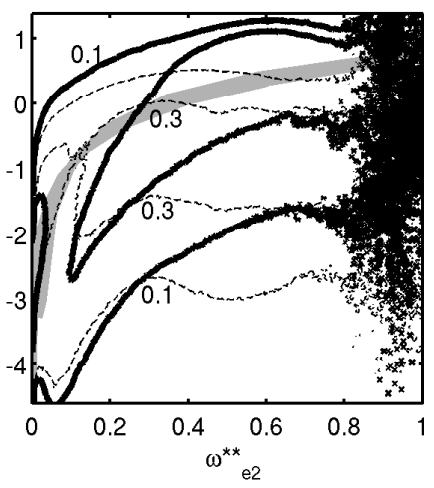

(i)

Figure 11: The conditional PDF, $P\left(\psi_{i}^{+} \mid \omega^{* *}\right)$, of scalar gradient normal component $\psi_{1}^{+}$(solid line) and tangential component $\psi_{2}^{+}$(dashed line) for the premixed case (a, d, g), Case A (b, e, h), and Case B (c, f, i). (a-c): PDFs for the DNS fields, (d-f): for the synthesised $\omega_{e 1}$ and $c_{\text {PLIF }}$ fields, and (g-i): for the synthesised $\omega_{e 2}$ and $c_{\mathrm{PLIF}}$. The grey line is the respective laminar flame solution, $\left(\psi_{\mathrm{lam}}^{+}, \omega^{* *}\right)$. 
about 0.3 in the Case B. The difference in the behaviour of the PDFs for the normal and tangential components in the MILD cases is not as big as for the premixed case, suggesting that the MILD combustion may be distributed-like, which is because of the presence of copious interaction of reaction zones. The distributed reaction zones are expected to have similar level of scalar gradients in both the normal and tangential directions, which is seen in Fig. 11 for regions with low reaction rate as noted above. This can result from flame thickening [50] or extensive and copious interaction of flamelets. There is, however, a positive correlation between the normal component and reaction rate over the almost entire range of $\omega_{c_{T}}^{* *}$ as shown in Figs. $11 \mathrm{~b}$ and $11 \mathrm{c}$. Such a behaviour is flamelet-like. The distributed-like reaction zones and the positive correlation between the reaction rate and scalar gradient suggests that the MILD combustion considered here has a "weak" flamelet behaviour, where both flamelet and non-flamelet characteristics are observed.

It is worth noting that the values of $\mathrm{Da}$ and $\mathrm{Ka}$ for the premixed and Case $\mathrm{A}$ are similar (see Table 2). Despite this, substantially different behaviour is seen for the Case A. The PDFs of both normal and tangential components have equally a long negative tail even for $\omega_{c_{T}}^{* *} \approx 0.7$ in the MILD cases unlike in the premixed case. Also, the probability to find $\psi_{1}^{+}>0$ is larger in the MILD case which is due to frequent straining thinning of the MILD reaction zones by turbulence. This probability is increased further in the Case B because of high dilution level. A close study of Figs. $11 \mathrm{~b}$ and $11 \mathrm{c}$ shows that the probability for $\psi_{1}^{+}>0$ is finite for $0.4 \leq \omega_{c_{T}}^{* *} \leq 0.8$. This probability decreases for $\omega_{c_{T}}^{* *}>0.8$ which is clearer for the Case B in Fig. 11c suggesting that the turbulent straining is influential only on the reaction zones with moderate reaction rates. The regions with top $20 \%$ of the 
reaction rate have strong thermo-chemical effects compared to turbulence. Since the MILD combustion includes interactions of reaction zones [8], there are reaction zones with smaller normal scalar gradient than the respective laminar value as one can observe in Fig. 11c. Also the PDFs of normal and tangential components in the MILD cases do not differ as much as in the premixed case suggesting that the scalar gradients in the MILD conditions are less directional dependent compared to the premixed combustion. These insights suggest that the current surface based description of turbulent combustion may be inadequate to describe turbulent MILD combustion and alternative methods need to be developed.

The conditional PDFs constructed using the synthesised LIF signals are shown in the second and third rows of Fig. 11 for all the cases studied. These PDFs are very similar to those obtained from the DNS data supporting the insights described above. Hence, it would be very helpful and informative to construct and study such PDFs using laser diagnostics involving $\mathrm{OH}$ and $\mathrm{CH}_{2} \mathrm{O}$ PLIF. However, full 3D PLIF is expensive and not an easy task at this time. Thus it is worth to study these PDFs obtained using 2D slices of the DNS data and the corresponding synthesised LIF signals to verify if the above insights can also be deduced from these PDFs.

The conditional PDFs constructed using 2D data are shown in Fig. 12 and the reaction rate is normalised using the respective maximum value observed in each 2D field. The corresponding laminar flame solution $\left(\psi_{\text {lam }}^{+}, \omega^{*}\right)$ is also shown (grey line) for comparison. There are some small differences in the PDF shape. However, the relative behaviour among the normal and tangential components, and laminar solution observed in Fig. 12 is very similar to those noted from Fig. 11 for the three dimensional data. Thus, these conditional PDFs constructed using 2D 


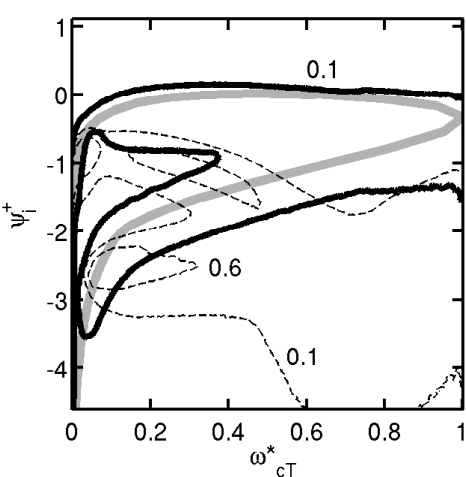

(a)

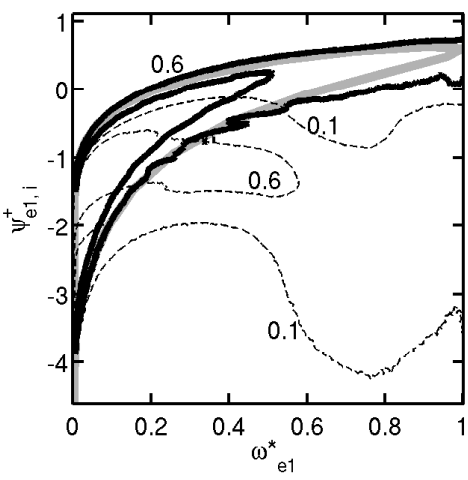

(d)

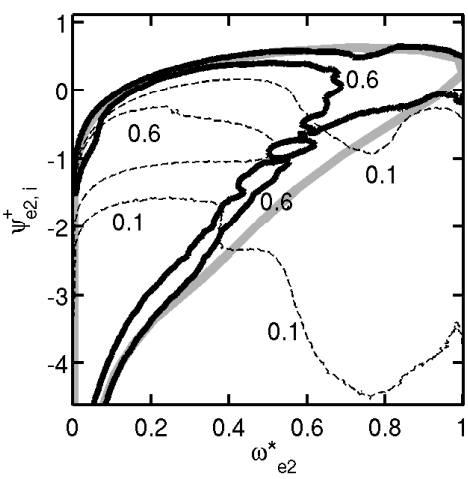

(g)

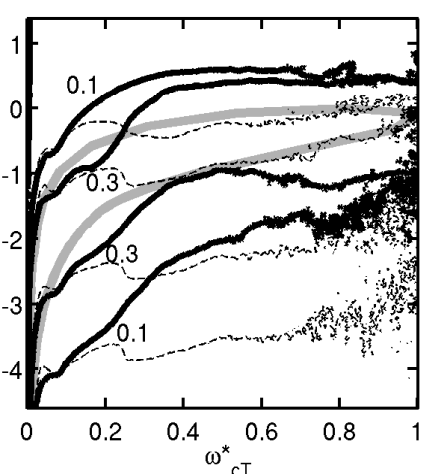

(b)

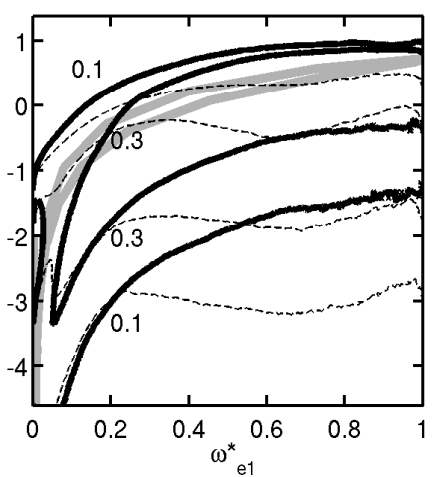

(e)

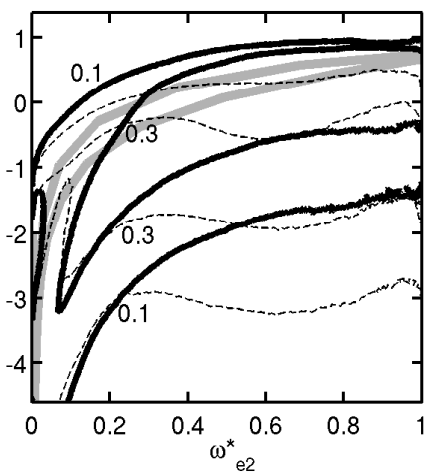

(h)

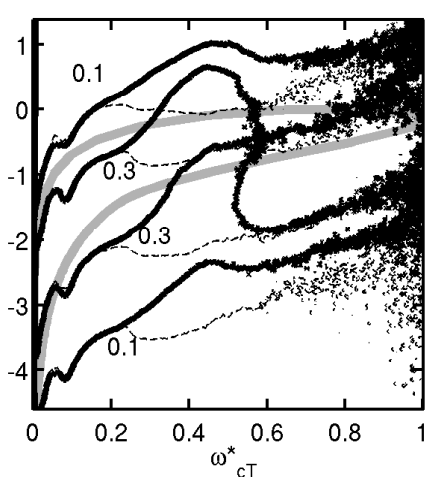

(c)

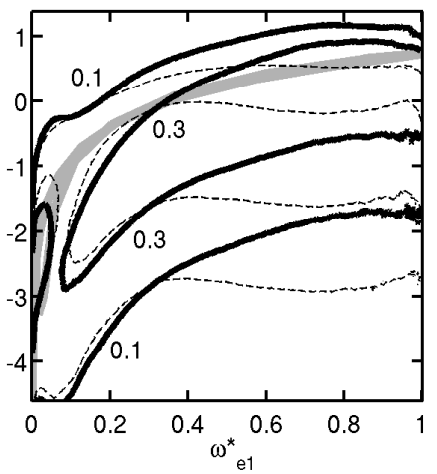

(f)

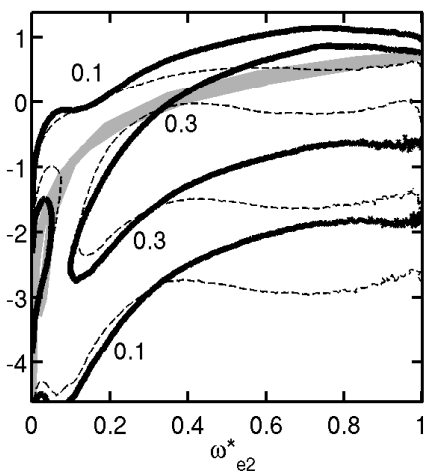

(i)

Figure 12: The conditional PDF, $P\left(\psi_{i}^{+} \mid \omega^{*}\right)$, of normal $\psi_{1}^{+}$(solid line) and tangential $\psi_{2}^{+}$(dashed line) components of 2D scalar gradient for the premixed case $(a, d, g)$, Case A (b, e, h), and Case B (c, f, i). (a-c): PDFs based on 2D slice of the DNS data, (d-f): based on synthesised $\omega_{e 1}$ and $c_{\mathrm{PLIF}}$, and (g-i): based on synthesised $\omega_{e 2}$ and $c_{\mathrm{PLIF}}$. The grey line is the respective laminar flame solution $\left(\psi_{\text {lam }}^{+}, \omega^{*}\right)$. 
slices of three dimensional field have adequate information to gain insights into the flamelet or non-flamelet behaviour of reaction zones in turbulent MILD and premixed combustion.

\section{Conclusions}

Three-dimensional DNS data of turbulent MILD and premixed combustion has been studied to investigate the characteristics of reaction zones. The combustion kinetics in the DNS were represented using a skeletal mechanism for methane-air combustion and two dilution levels were considered for the MILD combustion. Generally, MILD or "flameless" combustion is expected to involve relatively moderate and spatially uniform chemical activities. However, the analyses show that there are indeed thin regions with strong reaction rate leading to large scalar gradients implying the presence of flamelets. These flamelets interact with one another and this interaction is very frequent in space and time leading to substantial thickening of the flamelets and enhanced reaction rates. The characteristics of these reaction zones are investigated here by studying the scalar gradients of a reaction progress variable $c_{T}$. Also, LIF signals of $\mathrm{OH}, \mathrm{CH}_{2} \mathrm{O}$ and $\mathrm{CHO}$ are synthesised from the DNS data to construct a reaction progress variable field, $c_{\text {PLIF }}$, its gradient, and heat release rate information that could be obtained in a physical experiments. These synthesised signals are analysed to verify if the scientific insights deduced from them corroborate well with those from the actual scalar and reaction rate fields.

The heat release rate field obtained using the synthesised LIF signals represents the actual field from the DNS for both the MILD and premixed combustion well. This is well known for the premixed combustion and used routinely to study 
premixed combustion using laser diagnostics, but this had not been investigated for the MILD combustion. A good correlation between the actual and synthesised heat release rate fields observed in this study suggests that the LIF signals of $\mathrm{OH}$ and $\mathrm{CH}_{2} \mathrm{O}$ can be used for the MILD combustion of $\mathrm{CH}_{4}$ also. The spatial inhomogeneity in the mixture composition and its temporal variation in the MILD combustion does not seem to impart undue influences on the applicability of LIF technique to detect MILD reaction zones. However, using only $\mathrm{OH}$ or $\mathrm{CH}_{2} \mathrm{O}$ or CHO may be inadequate to study MILD combustion characteristics. Specifically, the progress variable field, $c_{\text {PLIF }}$, defined using the synthesised OH-LIF signal does not correlate quite well with $c_{T}$ in MILD combustion, except for high heat releasing regions. Although, a range of $c_{\mathrm{PLIF}}$ is observed for a given $c_{T}$ value, the iso-contours of these two variables are observed to be parallel in predominant regions of the MILD combustion. Thus the directions of $\nabla c_{\mathrm{PLIF}}$ and $\nabla c_{T}$ are quite close. These gradients are then projected onto local flame normal and tangential directions. The flame is identified using the reaction rate (both DNS and deduced field using the synthesised LIF signals) gradient to avoid multiply connected surfaces in MILD combustion. The reaction rate and projected scalar gradients are used to construct the PDFs of these gradients conditioned on the reaction rate.

The conditional PDFs show that the premixed case considered here has a typical flamelet combustion characteristics; stronger normal gradient compared to tangential gradients. However, the PDFs for MILD cases are markedly different. The PDFs of the normal component is broad in the MILD cases compared to the premixed case. The PDFs shows that the tangential gradient in the MILD combustion is not as small as in the premixed case. This suggests that the reaction zones in the MILD combustion is non-flamelet like. However, the normal gra- 
dient is relatively stronger than the tangential gradients in the MILD combustion also, implying the presence of thin reaction zones. Indeed these zones are observed using contours of reaction rate. These reaction zones interact often, which led to a relatively weak flamelet like combustion, in a broader sense, in the MILD cases. These results demonstrate that the so-called flameless combustion is not an idealised homogeneous reactive mixture but has common features of conventional combustion while having distinctive characteristics.

The $\mathrm{OH}-$ and $\mathrm{CH}_{2} \mathrm{O}$-LIF signals synthesised from the DNS data also corroborate these insights. The characteristics of reaction zones in turbulent combustion deduced using scalar gradients are expected to be more robust than those from the scalar field itself. It would be worthwhile to investigate these conditional PDFs that could be obtained from laser diagnostics of premixed and MILD combustion.

\section{Acknowledgment}

The financial supports of Nippon Keidanren, Cambridge Overseas Trust and EPSRC are acknowledged. The direct simulations were made using the facilities of HECToR, the UK's national high-performance computing service, which is provided by UoE HPCx Ltd at the University of Edinburgh, Cray Inc and NAG Ltd, and funded by the Office of Science and Technology through EPSRCs High End Computing Programme.

[1] J. A. Wünning, J. G. Wünning, Prog. Energy Combust. Sci. 23 (1997) 81-94.

[2] M. Katsuki, T. Hasegawa, Proc. Combust. Inst. (1998) 3135-3146.

[3] A. Cavaliere, M. de Joannon, Prog. Energy Combust. Sci. 30 (2004) 329366. 
[4] T. Plessing, N. Peters, J. G. Wünning, Proc. Combust. Inst. (1998) 31973204.

[5] I. B. Özdemir, N. Peters, Exp. Fluids 30 (2001) 683-695.

[6] B. B. Dally, E. Riesmeier, N. Peters, Combust. Flame 137 (2004) 418-431.

[7] C. Duwig, B. Li, M. Aldén, Combust. Flame 159 (2012) 306-316.

[8] Y. Minamoto, T. D. Dunstan, N. Swaminathan, R. S. Cant, Proc. Combust. Inst. 34 (2013) 3231-3238.

[9] E. P. Hassel, S. Linow, Measurement Science and Technology 11 (2000) R37.

[10] Q.-V. Nguyen, P. H. Paul, Proc. Combust. Inst. (1996) 357-364.

[11] P. H. Paul, H. N. Najm, Proc. Combust. Inst. (1998) 43-50.

[12] R. Balachandran, B. Ayoola, C. Kaminski, A. Dowling, E. Mastorakos, Combustion and Flame 143 (2005) 37 - 55.

[13] A. Fayoux, K. Zhringer, O. Gicquel, J. Rolon, Proc. Combust. Inst. 30 (2005) $251-257$.

[14] M. Tanahashi, S. Murakami, G. M. Choi, Y. Fukuchi, T. Miyauchi, Proc. Combust. Inst. 30 (2005) 1665-1672.

[15] J. Kiefer, Z. S. Li, T. Seeger, A. Leipertz, M. Aldén, Proc. Combust. Inst. 32 (2009) 921-928. 
[16] M. Richter, R. Collin, J. Nygren, M. Aldén, L. Hildingsson, B. Johansson, JSME International Journal Series B Fluids and Thermal Engineering 48 (2005) 701-707.

[17] Z. Li, B. Li, Z. Sun, X. Bai, M. Aldn, Combust. Flame 157 (2010) 1087 1096.

[18] M. Röder, T. Dreier, C. Schulz, Proceedings of the Combustion Institute 34 (2013) $3549-3556$.

[19] H. N. Najm, P. H. Paul, C. J. Mueller, P. S. Wyckoff, Combust. Flame 113 (1998) 312-332.

[20] H. N. Najm, O. M. Knio, P. H. Paul, P. S. Wyckoff, Comb. Sci. Technol. 140 (1998) 369-403.

[21] P. R. Medwell, Laser diagnostics in MILD combustion, Ph.D. thesis, The University of Adelaide, Adelaide, Australia, 2007.

[22] E. Abtahizadeh, J. van Oijen, P. de Goey, Combust. Flame 159 (2012) 21552165 .

[23] G. Sorrentino, D. Scarpa, A. Cavaliere, Proc. Combust. Inst. 34 (2013) 3239-3247.

[24] A. Cavaliere, M. de Joannon, R. Ragucci, in: D. D. Rankin (Ed.), Lean Combustion, Elsevier, 2008, pp. 55-94.

[25] P. Sabia, M. de Joannon, G. Sorrentino, G. Cozzolino, A. Cavaliere, International Journal of Energy for a Clean Environment 11 (2010) 21-34. 
[26] M. de Joannon, P. Sabia, G. Cozzolino, G. Sorrentino, A. Cavaliere, Combust. Sci. Technol. 184 (2012) 1207-1218.

[27] T. Poinsot, S. Lele, J. Comput. Phys. 101 (1992) 104-129.

[28] K. Jenkins, R. Cant, in: Knight, Sakell (Eds.), Proc. Second AFOSR Conf. on DNS and LES, Rutgers University, Kluwer Academic Publishers, 1999, pp. 192-202.

[29] R. S. Cant, in: Technical Report CUED/A-THERMO/TR67, Cambridge University Engineering Department, 2012.

[30] T. D. Dunstan, N. Swaminathan, K. N. C. Bray, R. S. Cant, Flow Turbulence Combust. 87 (2011) 237-259.

[31] T. D. Dunstan, N. Swaminathan, K. N. C. Bray, J. Fluid Mech. 709 (2012) $191-222$.

[32] M. D. Smooke, V. Giovangigli, in: M. D. Smooke (Ed.), Reduced kinetic mechanisms and asymptotic approximations for methane-air flames., volume 384, Springer Verlag, New York, 1991, pp. 1-28.

[33] G. K. Batchelor, A. A. Townsend, Proc. Roy. Soc. Lond. A 194 (1948) 527543.

[34] R. S. Rogallo, NASA TM (1981) 81315.

[35] V. Eswaran, S. B. Pope, Phys. Fluids 31 (1987) 506-520.

[36] R. W. Bilger, S. H. Stårner, R. J. Kee, Combust. Flame 80 (1990) 135-149. 
[37] R. J. Kee, J. F. Grcar, M. Smooke, J. A. Miller, PREMIX: A Fortran program for modeling steady one-dimensional flames, Sandia National Laboratories, 1985. SAND85-8240.

[38] P. Habisreuther, F. C. C. Galeazzo, C. Prathap, N. Zarzalis, Combust. Flame DOI:10.1016/j.combustflame.2013.06.023 (2013).

[39] Y. Suzukawa, S. Sugiyama, Y. Hino, M. Ishioka, I. Mori, Energy Convers. Mgmt 38 (1997) 1061-1071.

[40] A. Buschmann, F. Dinkelacker, T. Schäfer, J. Wolfrum, Proc. Combust. Inst. (1996) 437-445.

[41] Y. C. Chen, N. Peters, G. A. Schneemann, N. Wruck, U. Renz, M. S. Mansour, Combust. Flame 107 (1996) 223-244.

[42] S. Pfadler, A. Leipertz, F. Dinkelacker, Combust. Flame 152 (2008) 616631.

[43] E. Oldenhof, M. J. Tummers, E. H. van Veen, D. J. E. M. Roekaerts, Combust. Flame 158 (2011) 1553-1563.

[44] N. Peters, Turbulent combustion, Cambridge University Press, Cambridge, UK, 2000.

[45] T. Poinsot, S. Candel, A. Trouvé, Prog. Energy Combust. Sci. 21 (1996) $531-576$.

[46] R. Puri, M. Moser, R. J. Santoro, K. C. Smyth, Proc. Combust. Inst. 24 (1992) $1015-1022$. 
[47] A. Cessou, D. Stepowski, Combustion Science and Technology 118 (1996) $361-381$.

[48] R. Schiessl, U. Maas, A. Hoffmann, J. Wolfrum, C. Schulz, Applied Physics B 79 (2004) 759-766.

[49] C. C. Rasmussen, S. K. Dhanuka, J. F. Driscoll, Proc. Combust. Inst. 31 (2007) $2505-2512$.

[50] M. de Joannon, G. Sorrentino, A. Cavaliere, Combust. Flame 159 (2012) $1832-1839$. 